\title{
Dimension of invariant measures for maps with exponent zero
}

\author{
F. LEDRAPPIER AND M. MISIUREWICZ \\ Laboratoire de Probabilités, Université Paris VI, T56; 6, Place Jussieu, \\ 75230 Paris Cedex 05, France; Instytut Matematyki, Universytet Warszawski, \\ PKiN IXp, 00.901 Warszawa, Poland
}

(Received 17 September 1984 and revised 20 February 1985)

\begin{abstract}
We give examples of maps of the interval with zero entropy for which the continuous invariant measure has no dimension, and we prove a dimension property for maps lying in the stable manifold of Feigenbaum's fixed points.
\end{abstract}

\section{Introduction}

Chaotic behaviour of dynamical systems is sometimes reflected by the geometrical intricacy of invariant sets. It has been noticed recently that this complexity could be well described by dimensional quantities and particularly by the dimension of the invariant ergodic measures. We are interested here in one-dimensional systems, obtained by iteration of a smooth map of an interval. If $f$ is of class $C^{2}$, and if the exponent is negative, the measure is carried by a periodic sink. If the exponent is positive and if $f$ and $f^{\prime}$ are piecewise monotonic, then the dimension is given by the following formula

$$
\lim _{\eta \rightarrow 0} \frac{\log \mu([x-\eta, x+\eta])}{\log \eta}=\frac{h_{\mu}(f)}{\int \log \left|f^{\prime}\right| d \mu} \quad \mu \text {-a.e. }
$$

(see the appendix).

We want to discuss here one case when the exponent is zero, namely the case of unimodal maps with the same topological type as Feigenbaum's map (see [4] for the general background and [10] where the dynamic is described).

These maps possess a unique non-atomic invariant ergodic probability measure, which will be the one we shall consider.

Our first result is that for any $r<+\infty$ we can construct such a map of class $C^{r}$ for which the limit in $(*)$ does not exist. However, our construction does not give a $C^{\infty}$ map with that property.

The second result is that such a limit exists as soon as the map lies on the stable manifold of some fixed point of the Feigenbaum's renormalization operator. This dimension is also a 'universal' constant, i.e. depends only on the fixed point itself, as predicted by some numerical evidence [6]; for the analytic Feigenbaum fixed point, Y. Lévy communicated to us the following approximate value given by our formula in theorem $3: 0.517 \ldots$ 
This work was done during a visit of the first author to Warsaw and he would like to acknowledge the hospitality and support of the Polish Academy of Sciences. This research was supported in part by NSF Grant No. 8120790 and AFOSR Grant No. AFOSR-83-0265.

\section{Statement of results}

The precise result we shall prove is the following:

THEOREM 1. Let $A^{-1 / r-1}<B \leq C<A / A+1$, for some integer $r>1$, or let $r=1, A>1$ and $0<B \leq C<A / A+1$. Then there exists a $C^{r}$-map $f:[0,1] \rightarrow[0,1]$ and an $f$ invariant ergodic probability measure $\mu$, such that the metric entropy of $([0,1], f, \mu)$ is 0 and for $\mu$-a.e. $x$ in $[0,1]$ :

and

$$
\liminf _{\eta \rightarrow 0} \frac{\log \mu([x-\eta, x+\eta])}{\log \eta}=\frac{\log 2}{\log (\sqrt{A / B})}
$$

$$
\limsup _{\eta \rightarrow 0} \frac{\log \mu([x-\eta, x+\eta])}{\log \eta}=\frac{\log 2}{\log (\sqrt{A / C})}
$$

We say that the measure $\mu$ on $[0,1]$ has dimension $d$ when the limit

$$
\lim _{\eta \rightarrow 0} \frac{\log \mu([x-\eta, x+\eta])}{\log \eta}=d
$$

for $\mu$ almost all $x$.

COROLlary 1. For every integer $r \geq 1$, there exists a $C^{r}$-map $f:[0,1] \rightarrow[0,1]$ and an $f$ invariant ergodic probability measure with no dimension.

COROLlaRY 2. For every integer $r \geq 1$, there exists a positive number $d_{r}$, such that for all $0<d<d_{r}$, there exists a $C^{r}$-map $f:[0,1] \rightarrow[0,1]$ and an $f$-invariant probability measure $\mu$ with entropy 0 and dimension $d$. We have

$$
d_{1}=1, \quad d_{2}=\frac{\log 4}{\log (2+\sqrt{5})}, \cdots \quad d_{r} \rightarrow 0 \quad \text { as } r \rightarrow+\infty
$$

and $d_{r}$ is of order $\log 4 / \log r$ as $r \rightarrow+\infty$.

We also remark that by considering exactly the example from [11], we have

THEOREM 2. There exist a $C^{\infty}$-map $f:[0,1] \rightarrow[0,1]$ and an $f$-invariant non-atomic ergodic probability measure with dimension, entropy and exponent all 0 .

All the maps constructed here are unimodal but far from being symmetric. On symmetric maps, we can define a doubling transformation. With the notation of [4], let $\mathbb{P}$ denote the space of maps $f:[-1,+1] \rightarrow[-1,+1]$ such that (a) $f$ is even, $f(0)=1$; (b) $f$ is $C^{1}, x f^{\prime}(x)<0$ if $x \neq 0$. Define for $f$ in $\mathbb{P}$,

$$
a(f)=-f(1), \quad b(f)=f(a(f)) .
$$


We define the doubling transformation $T$ on the subset of $\mathbb{P}$ where $0<a(f)<b(f)$ and $f(b(f))<a(f)$ by

$$
T f(x)=-\frac{1}{a(f)} f^{2}(-a(f) x) .
$$

We shall consider the set $\mathscr{F}$ of $g \in \mathbb{P}$ such that $T g=g$ and $1 / \sqrt{\left|g^{\prime}\right|}$ is convex on $[-1,0)$ and $(0,1]$. If $g \in \mathscr{F}$, then we use $W^{s}(g)$ to denote the set of maps $f$ in $\mathbb{P}$ such that the operator $T^{n} f=T\left(T^{n-1} f\right)$ is inductively defined for all $n \geq 0$ and

$$
\lim _{n \rightarrow \infty} \max \left(\sup _{x}\left|\left(T^{n} f\right)(x)-g(x)\right|, \sup _{x}\left|\left(T^{n} f\right)^{\prime}(x)-g^{\prime}(x)\right|\right)=0 .
$$

We define $W^{s}=\bigcup_{g \in G} W^{s}(g)$.

It is known that in the subset of $\mathbb{P}$ made of maps which are analytic in $|x|^{1+\varepsilon},(\varepsilon$ small or $\varepsilon=1$ ), $W^{s}$ is a submanifold of codimension 1 ([3], [5], [8]). If $f \in W^{s}$, then the kneading invariant of $f$ is the same as of $g$ and therefore there exists an $f$-invariant ergodic non-atomic probability measure $\mu_{f}$ with entropy 0 (see [4], [10]).

TheOREM 3. Let $f \in W^{s}(g)$, where $g \in \mathscr{F}$. Then $\mu_{f}$ has dimension

$$
\frac{\log 2}{\gamma(g)+\log \frac{1}{a(g)}},
$$

where $\gamma(g)=\int_{[b(g), 1]} \log \left|g^{\prime}\right| d \mu_{g}$.

A similar result has been obtained independently by K. M. Khanin, J. G. Sinai and H. B. Vul [7]. Although they use thermodynamical formalism, their ideas seem to be close to ours.

Corollary 3. Let $f \in W^{s}$, then $\operatorname{dim} \mu_{f}>0$ and $\int \log \left|f^{\prime}\right| d \mu_{f}=0$.

Finally let us recall that the dimension we are considering here is the minimal value of the Hausdorff dimension of sets of $\mu_{f}$-measure 1, but that the Hausdorff dimension or the capacity of the support of $\mu_{f}$ might be bigger. This occurs, for instance, in the example from [11] (compare the result of [11] and theorem 2).

\section{Construction of the map $f$ in theorem 1}

The construction follows closely the one from [11]. Fix a positive integer $r$ and positive numbers $A, B, C$ such that either $r \geq 2$ and $A^{-1 /(r-1)}<B \leq C<A /(A+1)$, or $r=1, A>1$ and $0<B \leq C<A /(A+1)$. In the first case, we have $1<A+1<$ $A^{1+(1 /(r-1))}$, and thus $A>1$ also in this case.

Choose a sequence $\theta_{n}(n=1,2, \ldots)$ with $\theta_{1}=1, B \leq \theta_{n+1} / \theta_{n} \leq C$. Notice that since $C<1, \theta_{n}$ decreases towards 0 . We start by defining two sequences of points of the interval $[0,1]$ :

$$
0=a_{2}<b_{2}<a_{4}<b_{4}<a_{6}<b_{6}<\cdots<b_{7}<a_{7}<b_{5}<a_{5}<b_{3}<a_{3}<b_{1}<a_{1}=1
$$

by setting

$$
\left|L_{n}\right|=\left|a_{n}-b_{n}\right|=\frac{\theta_{n+1}}{A}
$$




$$
\left|M_{n}\right|=\left|a_{n+2}-b_{n}\right|=\theta_{n}-\theta_{n+1}-\frac{\theta_{n+1}}{A} ;
$$

( $L_{n}$ and $M_{n}$ are the intervals with end points $a_{n}, b_{n}$ and $b_{n}, a_{n+2}$ respectively). We have

$$
\frac{\left|M_{n}\right|}{\theta_{n}} \geqslant 1-C \frac{(A+1)}{A}>0 .
$$

Since

$$
\sum_{n=1}^{\infty}\left\{\left|L_{n}\right|+\left|M_{n}\right|\right\}=\sum_{n=1}^{\infty}\left(\theta_{n}-\theta_{n+1}\right)=\theta_{1}=1
$$

we get that all points with even indices lie to the left of all points with odd indices and there exists a common $\operatorname{limit} c=\lim a_{n}=\lim b_{n}$.

Set $\mu_{n}=A^{n-1}$. We now define $f$ :

$$
\begin{aligned}
& f(c)=1, \\
& f\left(a_{n}\right)=1-\gamma_{n}, \quad \text { where } \gamma_{n}=\theta_{n} / A^{n-1}, \\
& f\left(b_{n}\right)=1-\delta_{n}, \quad \text { where } \delta_{n}=\left(\theta_{n}-\theta_{n+1}\right) / A^{n-1} .
\end{aligned}
$$

We have $1=\gamma_{1}>\delta_{1}>\gamma_{2}>\delta_{2}>\cdots \rightarrow 0\left(\delta_{n}>\gamma_{n+1}\right.$, because $\left[\left(\theta_{n}-\theta_{n+1}\right) / A^{n-1}\right]-$ $\left.\left(\theta_{n+1} / A^{n}\right)=\left|M_{n}\right| / A^{n-1}\right)$ and we define $f$ as a linear map on each interval $L_{n}$. The slope of $f$ on $L_{n}$ is denoted by $\lambda_{n}$. We have

$$
\lambda_{n}=\left|\frac{f\left(b_{n}\right)-f\left(a_{n}\right)}{b_{n}-a_{n}}\right|=\frac{\gamma_{n}-\delta_{n}}{\left|L_{n}\right|}=A^{2-n}=\frac{\mu_{n+1}}{\mu_{n}^{2}} .
$$

Since $[0,1]=\{c\} \cup \bigcup_{n \geq 1} L_{s} \cup \bigcup_{n \geq 1} M_{n}$, it remains to define $f$ on the gaps $M_{n}$.

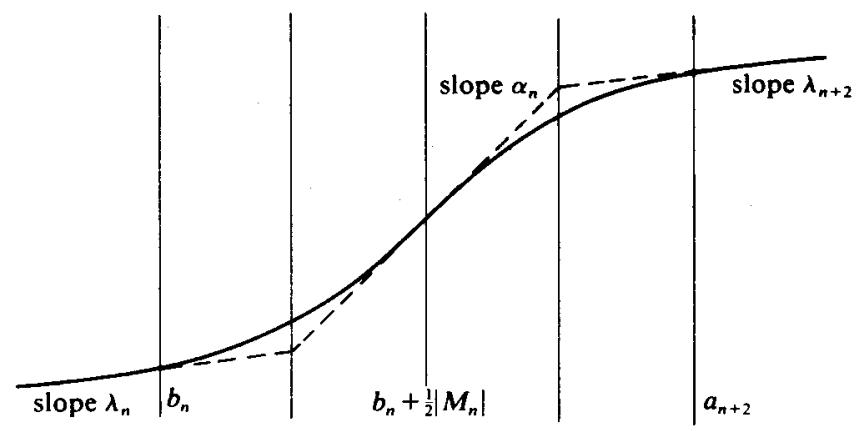

FIGURE 1

Write

$$
\omega_{n}=\frac{f\left(a_{n+2}\right)-f\left(b_{n}\right)}{a_{n+2}-b_{n}}, \quad \alpha_{n}=2 \omega_{n}-\frac{1}{2}\left(\lambda_{n}+\lambda_{n+2}\right)
$$

(Figure 1 is in the case $n$ even).

For $n$ even we put

$$
\begin{gathered}
f\left(b_{n}+\frac{1}{2}\left|M_{n}\right|\right)=f\left(b_{n}\right)+\frac{1}{4}\left|M_{n}\right|\left(\lambda_{n}+\alpha_{n}\right), \\
f^{\prime}\left(b_{n}+\frac{1}{2}\left|M_{n}\right|\right)=\alpha_{n}, \quad f^{(k)}\left(b_{n}+\frac{1}{2}\left|M_{n}\right|\right)=0 \quad \text { for } k>1,
\end{gathered}
$$


and we use the same function $g$ as in [11] to define $f$ separately on $\left[b_{n}, b_{n}+\frac{1}{2}\left|M_{n}\right|\right]$ and $\left[b_{n}+\frac{1}{2}\left|M_{n}\right|, a_{n+2}\right]$ as a $C^{\infty}$ function. For $n$ odd, we define $f$ analogously. This defines a map $f:[0,1] \rightarrow[0,1]$. The condition for $f$ to be of class $C^{1}$ is $\lim _{n \rightarrow 0} \max \left(\left|\lambda_{n}\right|,\left|\alpha_{n}\right|\right)=0$. It is satisfied since $\left|\lambda_{n}\right|=A^{2-n} \rightarrow 0$ as $n \rightarrow \infty$,

$$
\left|\omega_{n}\right|=\frac{f\left(a_{n+2}\right)-f\left(b_{n}\right)}{\left|M_{n}\right|}<\frac{\gamma_{n}}{\left|M_{n}\right|} \leq \frac{A^{2-n}}{(A+1)\left(\frac{A}{A+1}-C\right)}
$$

Thus $\left|\omega_{n}\right| \rightarrow 0$ as $n \rightarrow \infty$, and hence

$$
\left|\alpha_{n}\right| \leq 2\left|\omega_{n}\right|+\frac{1}{2}\left|\lambda_{n}\right|+\frac{1}{2}\left|\lambda_{n+2}\right| \rightarrow 0
$$

as $n \rightarrow+\infty$. If $r>1$, the condition for $f$ to be of class $C^{r}$ is

$$
\lim _{n \rightarrow \infty}\left(\frac{1}{2}\left|M_{n}\right|\right)^{1-r} \max \left(\left|\alpha_{n}-\lambda_{n}\right|,\left|\alpha_{n}-\lambda_{n+2}\right|\right)=0
$$

(see [11, relation (6), page 408]). We have

$$
\max \left(\left|\alpha_{n}-\lambda_{n}\right|,\left|\alpha_{n}-\lambda_{n+2}\right|\right) \leq\left|\alpha_{n}\right|+\left|\lambda_{n}\right|+\left|\lambda_{n+2}\right| \leq D A^{-n}
$$

for some constant $D$ independent of $n$. Hence,

$$
\left(\frac{1}{2}\left|M_{n}\right|\right)^{1-r} \max \left(\left|\alpha_{n}-\lambda_{n}\right|,\left|\alpha_{n}-\lambda_{n+2}\right|\right) \leq E A^{-n} \theta_{n}^{1-r}
$$

for some constant $E$ independent of $n$. Since $B A^{1 /(r-1)}>1$, we have

$$
A^{-n} \theta_{n}^{1-r} \leq\left(B A^{1 /(r-1)}\right)^{n(1-r)} \rightarrow 0 \quad \text { as } n \rightarrow \infty
$$

(remember that $1-r<0$ ).

Thus we have defined a $C^{r}$-map $f:[0,1] \rightarrow[0,1]$ which has the following properties, as in [11]. For $n \geq 1$, we set $h_{n}=f^{2^{n-1}}, g_{n}=\left.f^{2^{n-1}-1}\right|_{\left[f\left(a_{n}\right), 1\right]}$.

LEMMA 1. For every $n \geq 1$, we have: $h_{n}\left(a_{n}\right)=a_{n+1}, h_{n}\left(b_{n}\right)=a_{n+2}, h_{n}\left(a_{n+1}\right)=b_{n}$, $h_{n}(c)=a_{n}, g_{n}$ is linear and has slope $\mu_{n}, g_{n}$ preserves orientation if and only if $n$ is odd, $f$ is linear on $f^{i}\left(\left[f\left(a_{n}\right), 1\right]\right)$, for $i=0,1,2, \ldots, 2^{n-1}-2$.

Lemma 2. Put $K_{n}=\left[a_{n}, a_{n+1}\right]$ or $\left[a_{n+1}, a_{n}\right]$.

(a) The sequence $\left(K_{n}\right)_{n=1}^{\infty}$ is decreasing;

(b) $h_{n}\left(K_{n+1}\right)=L_{n}$;

(c) $h_{n}\left(K_{n}\right)=K_{n}$;

(d) the sets $f^{i} K_{n}, i=1,2,3, \ldots, 2^{n-1}$ are disjoint;

(e) $\left.f\right|_{f^{i} K_{n}}$ is linear for $i=1,2,3, \ldots, 2^{n-1}-1$;

(f) $f^{2^{n-1}+i}\left(K_{n+1}\right) \cap f^{i}\left(K_{n+1}\right)=\varnothing$ for $i=1,2,3, \ldots, 2^{n-1}$.

The proof of lemma 1 given in [11] is based on the equalities $\left|a_{n+1}-a_{n+2}\right|=$ $\mu_{n}\left|\gamma_{n}-\delta_{n}\right|,\left|a_{n+2}-b_{n}\right|=\mu_{n}\left|\delta_{n}-\gamma_{n+1}\right|,\left|a_{n+1}-a_{n}\right|=\mu_{n} \gamma_{n}$ and $\lambda_{n} \mu_{n}^{2}=\mu_{n+1}$. These equalities are also true with our values of $a_{n}, b_{n}, \delta_{n}, \gamma_{n}, \mu_{n}$ and $\lambda_{n}$. The proof of lemma 2(a)-(f) is the same as in [11]. Property (g) is not true here. Instead of lemma 2(g) and lemma 3 of [11], we have:

LEMMA 3. For each $i=1,2, \ldots, 2^{n-1}$ write $i=1+\sum_{k=1}^{n-1} \varepsilon_{k}(i) 2^{k-1}$ with $\varepsilon_{k}(i)=0$ or 1 . Put

$$
\zeta_{k}(0)=\frac{1}{A} \frac{\theta_{k+1}}{\theta_{k}}, \quad \zeta_{k}(1)=\frac{\theta_{k+1}}{\theta_{k}}
$$


Then for all $n \geqslant 1, i=1,2, \ldots, 2^{n-1}$,

$$
\left|f^{i}\left(K_{n}\right)\right|=\prod_{k=1}^{n-1} \zeta_{k}\left(\varepsilon_{k}(i)\right)
$$

Proof. We use induction on $n$. The conclusion of lemma 3 holds for $n=1$. Suppose now that it holds for $n=m$; we shall prove it for $n=m+1$.

Observe that for $i=1, \ldots, 2^{m-1}, f^{i}\left(K_{m}\right)$ is a disjoint (modulo end points) union of $f^{i}\left(K_{m+1}\right), f^{i}\left(L_{m}\right)$ (equal to $f^{2^{m-1}+i}\left(K_{m+1}\right)$ ) and a remaining gap $G_{i, m}$.

Observe also that $f^{i-1}$ is linear on $f\left(K_{m}\right)$ so that the lengths of these intervals are in the same proportions as:

$$
\frac{\left|f\left(K_{m+1}\right)\right|}{\left|f\left(K_{m}\right)\right|}=\frac{\gamma_{m+1}}{\gamma_{m}}=\frac{1}{A} \frac{\theta_{m+1}}{\theta_{m}} \text { and } \quad \frac{\left|f\left(L_{m}\right)\right|}{\left|f\left(K_{m}\right)\right|}=\frac{\gamma_{m}-\delta_{m}}{\gamma_{m}}=\frac{\theta_{m+1}}{\theta_{m}} .
$$

The induction follows clearly from these two observations.

Lemma 3 gives us information on the lengths of the intervals building the attractor. We also have information on the length of the gaps. Set

$$
\beta=\left(\frac{1}{C}-\frac{A+1}{A}\right)^{-1} \text {. }
$$

Clearly, $\beta>0$.

LEMMA 4. For all $m \geq 2$ and $i=1,2, \ldots, 2^{m-1}$

$$
\left|f^{i}\left(K_{m+1}\right)\right|<\left|f^{i}\left(L_{m}\right)\right| \leq \beta\left|G_{i, m}\right| \text {. }
$$

Proof. Observe again that by linearity of $\left.f^{i-1}\right|_{f\left(K_{m}\right)}$ the above inequalities are equivalent to

$$
\frac{\left|f\left(K_{m+1}\right)\right|}{\left|f\left(K_{m}\right)\right|}<\frac{\left|f\left(L_{m}\right)\right|}{\left|f\left(K_{m}\right)\right|} \leq \beta\left[1-\frac{\left|f\left(K_{m+1}\right)\right|}{\left|f\left(K_{m}\right)\right|}-\frac{\left.\mid f_{i}^{\prime} L_{m}\right) \mid}{\left|f\left(K_{m}\right)\right|}\right]
$$

i.e.

$$
\frac{1}{A} \frac{\theta_{m+1}}{\theta_{m}}<\frac{\theta_{m+1}}{\theta_{m}} \leq \beta\left[1-\left(1+\frac{1}{A}\right) \frac{\theta_{m+1}}{\theta_{m}}\right] .
$$

The first inequality holds and to prove the second inequality, we have to show that

$$
\beta^{-1} \leq \frac{\theta_{m}}{\theta_{m+1}}-\frac{A+1}{A} \text {. }
$$

This is equivalent to $1 / C \leq \theta_{m} / \theta_{m+1}$, which is clearly true.

We write $S=\bigcap_{n=1}^{\infty} \bigcup_{i=1}^{2^{n-1}} f^{i}\left(K_{n}\right)$. By lemma 3 and since $((A+1) / A) C<1, S$ is a Cantor set of zero Lebesgue measure. We define a probability measure $\mu$ on $S$ by assigning to every $f^{i}\left(K_{m}\right) i=1,2, \ldots, 2^{m-1}$ the measure $\mu\left(f^{i}\left(K_{m}\right)\right)=2^{1-m}$. The measure $\mu$ is clearly non-atomic and invariant.

For each $x \in S$, we define a 0 -1 sequence $\varepsilon(x)=\left(\varepsilon_{n}(x)\right)_{n=1}^{\infty}$ following the construction in lemma 3: if $x \in f^{i}\left(K_{n+1}\right), 1 \leq i \leq 2^{n}$, then $\varepsilon_{n}(x)=0$ if $i \leq 2^{n-1}, \varepsilon_{n}(x)=1$ if $i>2^{n-1}$. With the measure $\mu$, the sequence $\left(\varepsilon_{n}(x)\right)_{n=1}^{\infty}$ is an independent sequence of variables in $\{0,1\}$ with the same distribution $\left(\frac{1}{2}, \frac{1}{2}\right)$. 
It is also clear that $(S, \mu, f)$ is isomorphic by the map $x \rightarrow \varepsilon(x)$ to the system called adding-machine and thus is ergodic and has entropy 0 (see [10], [4]).

\section{Dimension of the invariant measure}

We shall now prove theorems 1 and 2 and discuss corollaries 1 and 2. From the properties of $f$ in $\S 2$, we get the following:

Lemma 5. For all $x \in S$,

$$
\begin{gathered}
\limsup _{n \rightarrow 0} \frac{\log m([x-\eta, x+\eta])}{\log \eta}=\limsup _{n \rightarrow \infty} \frac{-n \log 2}{\sum_{j=1}^{n} \log \zeta_{j}\left(\varepsilon_{j}(x)\right)} \\
\liminf _{\eta \rightarrow 0} \frac{\log m([x-\eta, x+\eta])}{\log \eta}=\liminf _{n \rightarrow \infty} \frac{-n \log 2}{\sum_{j=1}^{n} \log \zeta_{j}\left(\varepsilon_{j}(x)\right)}
\end{gathered}
$$

Proof. We set for $x \in f^{i}\left(K_{n+1}\right), i=1,2, \ldots, 2^{n}, \eta_{n}(x)=\left|f^{i}\left(K_{n+1}\right)\right|=\prod_{k=1}^{n} \zeta_{k}\left(\varepsilon_{k}(x)\right)$ (see lemma 3) and we fix $n_{0}$ such that $\beta<C^{-n_{0}}$. Clearly, the interval $\left[x-\eta_{n}(x)\right.$, $x+\eta_{n}(x)$ ] contains $f^{i}\left(K_{n+1}\right)$. For each $k<n$, there is $i(k)$ such that $1 \leq i(k) \leq 2^{k}$ and $x \in f^{i(k)}\left(K_{k+1}\right)$. By lemma 3, we have $\left|f^{i(k)}\left(K_{k+1}\right)\right| \geq C^{-(n-k)}\left|f^{i}\left(K_{n+1}\right)\right|$ and hence if $k \leq n-n_{0}$, then $\left|f^{i(k)}\left(K_{k+1}\right)\right| \geq \beta \eta_{n}(x)$. By lemma 4 , we see that if $k \leq n-n_{0}$, then $\left|G_{i(k), k}\right| \geq \eta_{n}(x)$. Consequently, the set $\left[x-\eta_{n}(x), x+\eta_{n}(x)\right] \cap S$ is contained in $f^{i\left(n-n_{0}\right)}\left(K_{n-n_{0}+1}\right)$ (remember that the sets $G_{i(k), k}$ are 'gaps' and hence are disjoint from $S$ ). Therefore,

$$
2^{-n} \leq \mu\left(\left[x-\eta_{n}(x), x+\eta_{n}(x)\right]\right) \leq 2^{-\left(n-n_{0}\right)} .
$$

Lemma 5 follows immediately from this relation, taking into account that for all $n \geq 1$,

$$
0<B A^{-1} \leq \frac{\eta_{n+1}(x)}{\eta_{n}(x)} \leq C<1 .
$$

We now can prove theorem 1: we have to consider the behaviour of the sequence $(1 / n) \sum_{j=1}^{n} \log \zeta_{j}\left(\varepsilon_{j}(x)\right)$ when $n \rightarrow+\infty$, knowing that $\varepsilon_{j}(x)$ is an independent stationary sequence, $\log \zeta_{j}(0)=\log \left(\theta_{j+1} / \theta_{j}\right)-\log A, \log \zeta_{j}(1)=\log \left(\theta_{j+1} / \theta_{j}\right)$. For $\mu$-a.e. $x \in S$, we have

$$
\begin{gathered}
\limsup _{n \rightarrow \infty} \frac{1}{n} \sum_{j=1}^{n} \log \zeta_{j}\left(\varepsilon_{j}(k)\right)=\lim \sup _{n} \frac{1}{n} \log \theta_{n}-\frac{1}{2} \log A, \\
\liminf _{n} \frac{1}{n} \sum_{j=1}^{n} \log \zeta_{j}\left(\varepsilon_{j}(x)\right)=\lim _{n} \inf \frac{1}{n} \log \theta_{n}-\frac{1}{2} \log A .
\end{gathered}
$$

If we choose the sequence $\theta_{n}$ such that

$$
B \leq \frac{\theta_{n+1}}{\theta_{n}} \leq C \text { and } \log B=\lim _{n} \inf \frac{1}{n} \log \theta_{n} \leq \limsup _{n} \frac{1}{n} \log \theta_{n}=\log C,
$$

we can apply the preceding construction and lemma 5 gives the conclusions of theorem 1.

We get corollary 1 as soon as we can find $A, B, C$ satisfying $A^{-1 /(r-1)}<B<C<$ $A /(A+1)$ if $r \geq 2$ and $0<B<C<A /(A+1), A>1$ if $r=1$. This is possible for any $r \geq 1$ by choosing $A$ large e:iough. 
To prove corollary 2 for $r=1$, we have to estimate the possible values of $\log 2 / \log (\sqrt{A} / x)$ where $A>1$ and $0<x<A /(A+1)$. For $A$ fixed, $x / \sqrt{A}$ takes all values in $(0, \sqrt{A} /(A+1))$ and hence $\log 2 / \log (\sqrt{A} / x)$ takes all values in $\left(0, \log 2 / \log ((A+1) / \sqrt{A})\right.$. We can choose $A$ arbitrarily close to 1 so that $d_{1}=$ $\lim _{A \rightarrow 1} \log 2 / \log ((A+1) / \sqrt{A})=1$. When $r>1$, we have to estimate the possible values of $x / \sqrt{A}$ for $A^{-1 /(r-1)}<x<A /(A+1)$. One can easily see for all $r>1$ that there exists a unique $A(r)>1$ such that

$$
A(r)^{-1 /(r-1)}=\frac{A(r)}{A(r)+1}
$$

and that $A^{-1 /(r-1)}<A /(A+1)$ for $A>1$ if and only if $A>A(r)$. Thus the possible values for $x / \sqrt{A}$ are

$$
\bigcup_{A>A(r)}\left(A^{-1 /(r-1)-1 / 2}, \frac{\sqrt{A}}{A+1}\right)=\left(0, \frac{\sqrt{A(r)}}{A(r)+1}\right) .
$$

Solving (**) for $r=2$ gives $A(2)=(1+\sqrt{5}) / 2$. Therefore

$$
d_{2}=\frac{\log 2}{\log \frac{A(2)+1}{\sqrt{A(2)}}}=\frac{\log 4}{\log (2+\sqrt{5})} \text {. }
$$

The equation $(* *)$ is equivalent to

$$
\frac{1}{r-1} A(r) \log A(r)=A(r) \log \left(1+\frac{1}{A(r)}\right) \text {. }
$$

Fix a number $\alpha, 0<\alpha \leq 1$. We have

and

$$
\lim _{r \rightarrow \infty} r^{\alpha} \log \left(1+\left(1 / r^{\alpha}\right)\right)=1
$$

$$
\lim _{r \rightarrow \infty} \frac{1}{r-1} r^{\alpha} \log r^{\alpha}=\left\{\begin{array}{rl}
+\infty & \text { if } \alpha=1 \\
0 & \text { if } \alpha<1
\end{array} .\right.
$$

Hence, if $\alpha<1$ and $r$ is sufficiently large, then $r^{\alpha}<A(r)<r$. We get for such an $r$ :

Hence

$$
\frac{\log 2}{\log \frac{r+1}{\sqrt{r}}} \leq d_{r} \leq \frac{\log 2}{\log \frac{r^{\alpha}+1}{r^{\alpha / 2}}}
$$

$$
1 \leq \liminf _{r \rightarrow+\infty} \frac{d_{r} \log \sqrt{r}}{\log 2} \leq \limsup _{r \rightarrow+\infty} \frac{d_{r} \log \sqrt{r}}{\log 2} \leq \frac{1}{\alpha} .
$$

This holds for all $\alpha \in(0,1)$ and hence $\lim _{r \rightarrow \infty} d_{r} \log \sqrt{r} / \log 2=1$.

Let us indicate also how we can estimate dimension and exponent in theorem 2. The example is exactly the map $f$ described in [11] and the dimension is computed as in theorem 1. Our lemma 3 is still valid, but with new functions $\zeta_{k}^{\prime}$ :

$$
\zeta_{k}^{\prime}(0)=\frac{k}{(k+1)^{2}}, \quad \zeta_{k}^{\prime}(1)=\frac{k}{k+1} .
$$

(Notice that in the last line of the proof of lemma 2 of [11], where the similar computations are made, there is a misprint: $1-a_{n+1}$ and $1-a_{n}$ should be replaced by $1-f\left(a_{n+1}\right)$ and $1-f\left(a_{n}\right)$, respectively.) 
For all $x$ in $S$, let us define

$$
\eta_{n}(x)=\left|f^{i}\left(K_{n+1}\right)\right|=\prod_{k=1}^{n} \zeta_{k}^{\prime}\left(\varepsilon_{k}(x)\right) .
$$

We again have $\left[x-\eta_{n}(k), x+\eta_{n}(x)\right] \supset f^{i}\left(K_{n+1}\right)$ and thus $\mu\left(\left[x-\eta_{n}(x), x+\eta_{n}(x)\right]\right) \geq$ $2^{-n}$. But now for $\mu$-a.e. $x$, we have

$$
\lim _{n \rightarrow+\infty} \frac{1}{n} \sum_{k=1}^{n} \log \zeta_{k}^{\prime}\left(\varepsilon_{k}(x)\right)=\lim _{n \rightarrow+\infty} \frac{1}{n} \sum_{k=1}^{n}\left(1-\varepsilon_{k}(x)\right) \log \frac{1}{k+1}=-\infty,
$$

and thus for all $t>1, \eta_{n}(x)<t^{-n}$ when $n$ is sufficiently large. Hence we have, for $\mu$-a.e. $x$ and for all $t>1$,

$$
\begin{aligned}
\limsup _{\eta \rightarrow 0} \frac{\log \mu([x-\eta, x+\eta])}{\log \eta} & =\frac{1}{\log t} \limsup _{n \rightarrow+\infty}-\frac{1}{n} \log \mu\left(\left[x-t^{-n}, x+t^{-n}\right]\right) \\
& \leq \frac{1}{\log t} \limsup _{n \rightarrow+\infty}-\frac{1}{n} \log \mu\left(\left[x-\eta_{n}(x), x+\eta_{n}(x)\right]\right) \\
& \leq \frac{\log 2}{\log t} .
\end{aligned}
$$

The dimension estimate follows by letting $t \rightarrow+\infty$.

Finally, since for all $n \geq 1,\left|f^{\prime}\right|=\lambda_{n}=(n+1) / n$ ! on $L_{n}$ and $\mu\left(L_{n}\right)=1 / 2^{n}$, an easy calculation gives $\int \log \left|f^{\prime}\right| d \mu=0$.

\section{Proof of theorem 3}

Let $f \in W^{s}$. We follow the description of the Cantor set $J$ and the invariant measure $\mu$ in [4, page 222], introducing minor modifications.

We recall that $f(0)=1, f(1)=-a(f), f(-a(f))=f(a(f))=b(f)$ and $0<f(b(f))<$ $a(f)<b(f)$. We define also $c(f)$ and $d(f)$ by $c(f)>0, f(c(f))=a(f)$ and $d(f)>0$, $f(d(f))=d(f)$. Then we set $K(f)=[b(f), 1], L(f)=[c(f), 1]$. We have

$$
f(K(f))=[-a(f), f(b(f))], \quad f(L(f))=[-a(f), a(f)]
$$

(see figure 2).

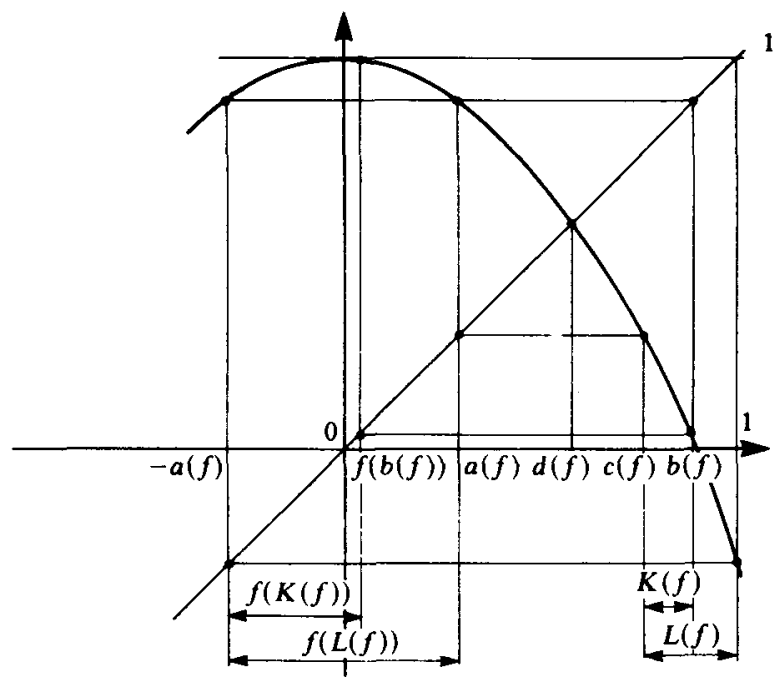

FIGURE 2 
Since $b(f)>a(f)$, we have $0<a(f)<d(f)<b(f)$. Hence, $c(f)>d(f)$, and since the fixed point $d(f)$ is repelling (see [4], [10]), $a(f)<c(f)<b(f)$. Hence, $L(f)>$ $K(f)$ and $L(f)$ is disjoint from $f(L(f))$.

Set $J_{1}(f)=K(f) \cup f(K(f))$ and $I_{1}(f)=L(f) \cup f(L(f))$. We define $\Sigma_{f}$ on $f(L(f)) \cup[d(f), 1] \supset I_{1}(f)$ by

$$
\Sigma_{f} x= \begin{cases}-\frac{x}{a(f)} & \text { if } x \in f(L(f)) \\ -\frac{f x}{a(f)} & \text { if } x \in[d(f), 1]\end{cases}
$$

Notice that $\Sigma_{f}$ maps both $f(L(f))$ and $L(f)$ diffeomorphically onto [-1,1], (see figure 3).

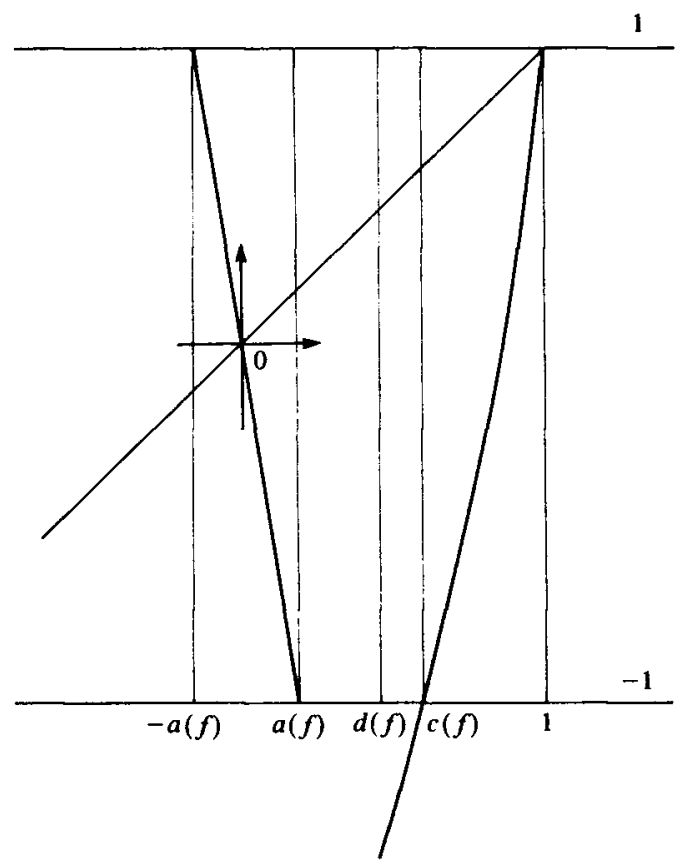

Figure 3

According to [4], the formula $J_{n}(f)=\Sigma_{f}^{-1}\left(J_{n-1}(T f)\right)$ defines a descending sequence of invariant closed sets. We denote $J(f)=\bigcap_{n \geq 1} J_{n}(f)$. Analogously, the formula $I_{n}(f)=\Sigma_{f}^{-1}\left(I_{n-1}(T f)\right)$ defines also a descending sequence of invariant closed sets. Since the set

$$
\Sigma_{f}\left(I(f) \backslash J_{1}(f)\right)=\left[-1,-\frac{f(b(f))}{a(f)}\right)=[-1,-a(T f))
$$

is disjoint from $I(T f)$, we have $\bigcap_{n \geq 1} I_{n}(f)=J(f)$ and therefore instead of considering the sets $J_{n}$ we may consider the sets $I_{n}$ (when applying lemma 6 ). 
Let $x \in J(f)$ and denote inductively $\sigma_{f}^{0} x=x, \sigma_{f}^{n} x=\Sigma_{T^{n-1} f}\left(\sigma_{f}^{n-1} x\right)$. Denote by $\varepsilon_{f}(x)=\left(\varepsilon_{n}(x)\right)_{n=0}^{\infty}$ the sequence such that

$$
\varepsilon_{n}(x)= \begin{cases}1 & \text { if } \sigma_{f}^{n} x \in L\left(T^{n} f\right), \\ 0 & \text { if } \sigma_{f}^{n} x \in\left(T^{n} f\right)\left(L\left(T^{n} f\right)\right) .\end{cases}
$$

By definition, $I_{n}(f)$ is made of $2^{n}$ intervals, each of them consisting of points with the same sequence $\varepsilon_{0}, \ldots, \varepsilon_{n-1}$. The invariant measure $\mu_{f}$ assigns to any such interval the measure $2^{-n}$.

Our idea is to replace the dynamics of $f$, which has Lyapunov exponent zero, by the dynamics of $\sigma_{f}$, which has positive Lyapunov exponent. If $f \in \mathscr{F}$, then $J(f)$ is $\Sigma_{f}$-invariant, $\mu_{f}$ is $\Sigma_{f}$-invariant, $\left.\Sigma_{f}\right|_{J(f)}$ is the full 2 -shift, $\left|\Sigma_{f}^{\prime}\right| \geq 1 / a(f)>1$, and therefore by $(*)$ and by the definition of $\Sigma_{f}$ we obtain the statement of theorem 3 . However, if $f \notin \mathscr{F}$, then the dynamics is time-dependent and we cannot use $(*)$.

Let $f \in W^{s}(g), g \in \mathscr{F}$. For $x \in J(f)$, denote by $\pi(x)$ the point in $J(g)$ with the same sequence associated: $\varepsilon_{f}(x)=\varepsilon_{g}(\pi(x))$. We shall use the closeness of $g$ and $T^{n} f$ to compare $x$ and $\pi(x)$.

LEMMA 6. For $n=1,2, \ldots$, let $M_{n}, N_{n}$ be closed subintervals of $[-1,1]$ such that $M_{n} \subset N_{n}$; let $\varphi_{n}$ be a diffeomorphism of $M_{n}$ onto $[-1,1]$ and $\psi_{n}$ a diffeomorphism of $N_{n}$ onto $\psi_{n}\left(N_{n}\right) \supset[-1,1]$; let

$$
\inf _{n \geq 1} \inf _{x \in N_{n}}\left|\psi_{n}^{\prime}(x)\right|=c>1,
$$

and let $\sup _{n \geq 1} \sup _{x \in M_{n}}\left|\varphi_{n}(x)-\psi_{n}(x)\right|$ be finite. Then for each $x$ such that all $\varphi_{n} \circ \cdots \circ \varphi_{1}$ are defined at $x$, the limit

$$
p(x)=\lim _{n \rightarrow \infty}\left(\psi_{1}^{-1} \circ \cdots \circ \psi_{n}^{-1}\right)\left(\left(\varphi_{n} \circ \cdots \circ \varphi_{1}\right)(x)\right)
$$

exists, and for each $k \geq 0$

$$
\left|\left(\varphi_{k} \circ \cdots \circ \varphi_{1}\right)(x)-\left(\psi_{k} \circ \cdots \circ \psi_{1}\right)(p(x))\right| \leq \frac{1}{c-1} \sup _{n>k} \sup _{x \in M_{n}}\left|\varphi_{n}(x)-\psi_{n}(x)\right| .
$$

Proof. For $m \geq n$, write $\Phi_{n}^{m}=\varphi_{m} \circ \cdots \circ \varphi_{n+1}, \Psi_{n}^{m}=\psi_{m} \circ \cdots \circ \psi_{n+1}$. Set

$$
\alpha_{k}=\sup _{n>k} \sup _{x \in M_{n}}\left|\varphi_{n}(x)-\psi_{n}(x)\right|, \quad \xi_{k}(t)=\frac{1}{c}\left(t+\alpha_{k}\right) .
$$

If $n>k$ and $y, z \in[-1,1]$ then

$$
\begin{aligned}
\left|\varphi_{n}^{-1}(y)-\psi_{n}^{-1}(z)\right| & \leq\left|\psi_{n}^{-1}\left(\psi_{n}\left(\varphi_{n}^{-1}(y)\right)\right)-\psi_{n}^{-1}\left(\varphi_{n}\left(\varphi_{n}^{-1}(y)\right)\right)\right|+\left|\psi_{n}^{-1}(y)-\psi_{n}^{-1}(z)\right| \\
& \leq \frac{1}{c}\left(\sup _{x \in M_{n}}\left|\psi_{n}(x)-\varphi_{n}(x)\right|+|y-z|\right) \leq \xi_{k}(|y-z|) .
\end{aligned}
$$

Since $\xi_{k}$ is increasing, we obtain by induction on $n$, for $m \geq n \geq k$,

$$
\left|\left(\Phi_{n}^{m}\right)^{-1}(y)-\left(\Psi_{n}^{m}\right)^{-1}(z)\right| \leq \xi_{k}^{m-n}(|y-z|) .
$$

In particular, for $y=z=\Phi_{0}^{m}(x)$ and $n=k$, we obtain

$$
\left|\Phi_{0}^{k}(x)-\left(\Psi_{k}^{m}\right)^{-1}\left(\Phi_{0}^{m}(x)\right)\right| \leq \xi_{k}^{m-k}(0)=\left(\frac{1}{c}+\frac{1}{c^{2}}+\cdots+\frac{1}{c^{m-k}}\right) \alpha_{k} \leq \frac{1}{c-1} \alpha_{k}
$$


Hence, if $m \geq k$ then we have

$$
\left|\left(\Psi_{0}^{k}\right)^{-1}\left(\Phi_{0}^{k}(x)\right)-\left(\Psi_{0}^{m}\right)^{-1}\left(\Phi_{0}^{m}(x)\right)\right| \leq \frac{1}{c^{k}(c-1)} \alpha_{k} .
$$

Therefore, since $\alpha_{k} \leq \alpha_{0}$ and $c>1$, if all $\Phi_{0}^{n}(x)$ are defined then the sequence $\left(\left(\Psi_{0}^{n}\right)^{-1}\left(\Phi_{0}^{n}(x)\right)\right)_{n=0}^{\infty}$ satisfies the Cauchy's condition, and consequently the limit (1) exists.

By (3) we have for $m>k$

$$
\left|\Phi_{0}^{k}(x)-\Psi_{0}^{k}\left(\left(\Psi_{0}^{m}\right)^{-1}\left(\Phi_{0}^{m}(x)\right)\right)\right|<\frac{1}{c-1} \alpha_{k}
$$

and by (1) we obtain (2).

LEMMA 7. If $f \in W^{s}(g)$, then

$$
\lim _{n \rightarrow \infty} \sup _{y \in J(f)}\left|\sigma_{f}^{n} y-\sigma_{g}^{n} \pi(y)\right|=0 .
$$

Proof. Assume first that $f$ is sufficiently close to $g$, and so are all $T^{n} f$. Then all $d\left(T^{n} f\right)$ are close to $d(g)$ and we may assume that $d\left(T^{n} f\right)<c(g)$ for all $n$. Fix $y \in J(f)$. We use lemma 6 with

Clearly, we have

$$
\begin{gathered}
M_{n}= \begin{cases}L(g) & \text { if } \varepsilon_{n-1}(y)=1, \\
g(L(g)) & \text { if } \varepsilon_{n-1}(y)=0 ;\end{cases} \\
N_{n}= \begin{cases}L(g) \cup L(f) & \text { if } \varepsilon_{n-1}(y)=1, \\
g(L(g)) \cup f(L(f)) & \text { if } \varepsilon_{n-1}(y)=0 ;\end{cases} \\
\varphi_{n}=\left.\Sigma_{g}\right|_{M_{n}}, \quad \psi_{n}=\left.\Sigma_{T^{n-1} f}\right|_{N_{n}} .
\end{gathered}
$$

$$
\lim _{n \rightarrow \infty} \sup _{x \in M_{n}}\left|\varphi_{n}(x)-\psi_{n}(x)\right|=0 .
$$

It remains to check that $(0)$ holds. If $T^{n} f$ is close to $g$ then $a\left(T^{n} f\right)$ is close to $a(g)$ and $a(g)<1$. Therefore, taking into account that $1 / \sqrt{\left|\left(T^{n} f\right)^{\prime}\right|}$ is convex for all $n$, it is enough to check that $\inf _{n \geq 1} \min \left(\left|\left(T^{n} f\right)^{\prime}(1)\right|,\left|\left(T^{n} f\right)^{\prime}\left(d\left(T^{n} f\right)\right)\right|\right)>a(g)$. The point $d\left(T^{n} f\right)$ is repelling and hence $\left|\left(T^{n} f\right)^{\prime}\left(d\left(T^{n} f\right)\right)\right| \geq 1$. Since $T g=g$, we get by differentiating this equation

and using convexity of $1 / \sqrt{\left|g^{\prime}\right|}$ and the (in-)equalities

$$
\left|g^{\prime}(1)\right|=\lim _{x \rightarrow 0} \frac{g^{\prime}(x)}{g^{\prime}(a(g) x)},
$$

$$
\lim _{x \rightarrow 0} \frac{1}{\sqrt{\left|g^{\prime}(x)\right|}}=+\infty \text { and } 0<a(g)<1,
$$

we obtain $\left|g^{\prime}(1)\right| \geq 1$. Therefore, if all $T^{n} f$ are sufficiently close to $g$, then inf $_{n \geq 1}\left|\left(T^{n} f\right)^{\prime}(1)\right|>a(g)$. Hence, the assumptions of lemma 6 are satisfied.

Since for $x=\pi(y)$ all maps $\varphi_{n} \circ \cdots \circ \varphi_{1}$ are defined at $x$, the limit (1) of lemma 6 exists. But all maps $\psi_{n} \circ \cdots \circ \psi_{1}$ are defined at $p(x)$ and such point is unique by (0). Therefore $p(x)=y$. By (2) and (5), and in view of the fact that $\left(\varphi_{k} \circ \cdots \circ \varphi_{1}\right)(x)=$ $\sigma_{g}^{k}(x)$ and $\left(\psi_{k} \circ \cdots \circ \psi_{1}\right)(y)=\sigma_{f}^{k}(y)$, we obtain $(4)$. 
Now, if $f$ and $g$ (or some $T^{n} f$ and $g$ ) are not necessarily close, we replace $f$ by $T^{k} f$ and $x$ by $\sigma_{f}^{k} x$ for $k$ sufficiently large. Since $\sigma_{f}^{n} y=\sigma_{T_{f}^{n}}^{\bar{k}_{f}^{k}}\left(\sigma_{f}^{k} y\right)$ and $\sigma_{\mathrm{g}}^{n} \pi(y)=$ $\sigma_{g}^{n-k}\left(\sigma_{g}^{k} \pi(y)\right)$, we obtain (4) in this case too.

LeMMA 8. Let $f \in W^{s}(g)$ and let $\eta_{n}(x)$ be the length of the interval of $J_{n}(f)$ containing $x($ for $x \in J(f))$. Then for $\mu_{f}$-a.e. $x$

$$
\lim _{n \rightarrow \infty}-\frac{1}{n} \log \eta_{n}(x)=\log \frac{1}{a(g)}+\gamma(g) .
$$

Proof. Let us use $C_{f}\left(\varepsilon_{0}, \varepsilon_{1}, \ldots, \varepsilon_{n-1}\right)$ to denote the interval in $J_{n}(f)$ corresponding to the sequence $\left(\varepsilon_{0}, \varepsilon_{1}, \ldots, \varepsilon_{n-1}\right)$. By the definition of $\varepsilon_{f}$, we have

$$
\begin{aligned}
\eta_{n}(x) & =\left|C_{f}\left(\varepsilon_{0}(x), \ldots, \varepsilon_{n-1}(x)\right)\right| \\
& =\left|C_{T^{n-1} f}\left(\varepsilon_{n-1}(x)\right)\right| \cdot \prod_{i=0}^{n-2} \frac{\left|C_{T_{f}^{i} f}\left(\varepsilon_{i}(x), \ldots, \varepsilon_{n-1}(x)\right)\right|}{\left|C_{T^{i+1} f}\left(\varepsilon_{i+1}(x), \ldots, \varepsilon_{n-1}(x)\right)\right|} \\
& =\left|C_{T^{n-1} f}\left(\varepsilon_{n-1}(x)\right)\right| \cdot \prod_{i=0}^{n-2} \frac{\left|C_{T^{i} f}\left(\varepsilon_{i}(x), \varepsilon_{i+1}(x), \ldots, \varepsilon_{n-1}(x)\right)\right|}{\left|C_{T^{i} f}\left(0, \varepsilon_{i+1}(x), \ldots, \varepsilon_{n-1}(x)\right)\right|} \cdot a\left(T^{i} f\right) .
\end{aligned}
$$

By the mean value theorem, there exists $y_{i} \in C_{T^{i} f}\left(1, \varepsilon_{i+1}(x), \ldots, \varepsilon_{n-1}(x)\right)$, such that

$$
\frac{\left|C_{T_{f}}\left(1, \varepsilon_{i+1}(x), \ldots, \varepsilon_{n-1}(x)\right)\right|}{\left|C_{T^{i} f}\left(0, \varepsilon_{i+1}(x), \ldots, \varepsilon_{n-1}(x)\right)\right|}=\left|\left(T^{i} f\right)^{\prime}\left(y_{i}\right)\right|^{-1} \text {. }
$$

Thus,

$$
\log \eta_{n}(x)=\log \left|C_{T^{n-1} f}\left(\varepsilon_{n-1}(x)\right)\right|+\sum_{i=0}^{n-2} \log a\left(T^{i} f\right)-\sum_{i=0}^{n-2} \varepsilon_{i}(x) \log \left|\left(T^{i} f\right)^{\prime}\left(y_{i}\right)\right| .
$$

As $T^{i} f \rightarrow g$ and $\left(T^{i} f\right)^{\prime} \rightarrow g^{\prime}$, the sequence $\left((-1 / n) \log \eta_{n}(x)\right)_{n=1}^{\infty}$ has the same limit as the sequence

$$
\left(-\log a(g)+\frac{1}{n} \sum_{i=0}^{n-1} \varepsilon_{i}(x) \log \left|g^{\prime}\left(y_{i}\right)\right|\right)_{n=1}^{\infty}
$$

If $i$ is large enough then there is a constant $c>1$ such that for all $n>i$,

$$
\left|C_{T^{i} f}\left(\varepsilon_{i}, \ldots, \varepsilon_{n-1}\right)\right| \leq c^{-(n-i)}
$$

(cf. (0)). Hence, if $t>0$, then by lemma 7 and the above inequality, there exists $k$ such that if $i \geq k, n-i \geq k$ and $\varepsilon_{i}(x)=1$, then $\left|y_{i}-\sigma_{f}^{i} x\right|<t$ and $\left|\sigma_{f}^{i} x-\sigma_{y}^{i} \pi(x)\right|<t$. Since $b\left(T^{n} f\right) \rightarrow b(g)$ and $\left|g^{\prime}\right|$ is bounded and bounded away from 0 on $[d(g), 1]$, we see that the sequence $\left((-1 / n) \log \eta_{n}(x)\right)_{n=1}^{\infty}$ has the same limit as the sequence $\left(-\log a(g)+(1 / n) \sum_{i=0}^{n-1} \varepsilon_{i}(x) \log \left|g^{\prime}\left(\sigma_{g}^{i} \pi(x)\right)\right|\right)_{n=1}^{\infty}$.

Since $T g=g$, we have $\sigma_{g}^{i}=\Sigma_{g}^{i}$. The system $\left(J(g),\left.\Sigma_{g}\right|_{J(g)}, \mu_{g}\right)$ is isomorphic to the 1-sided Bernoulli shift $(1 / 2,1 / 2)$ (denote it $(\Omega, u, \nu)$ ) and this isomorphism is given by $\varepsilon_{g}$. If $\pi(x)=y$, then $\varepsilon_{i}(x)$ is the ith coordinate of $\varepsilon_{g}(y)$, and hence

$$
\varepsilon_{i}(x) \log \left|g^{\prime}\left(\sigma_{g}^{i} \pi(x)\right)\right|=G \circ u^{i},
$$

where for $\omega=\left(\omega_{j}\right)_{j=0}^{\infty} \in \Omega, G(\omega)=\omega_{0} \log \left|g^{\prime}\left(\varepsilon_{g}^{-1}(\omega)\right)\right|$. By the ergodic theorem, we have for $\nu$-almost every $\omega \in \Omega$,

$$
\lim _{n \rightarrow \infty} \frac{1}{n} \sum_{i=0}^{n-1}\left(G \circ u^{i}\right)(\omega)=\int_{\Omega} G d \nu
$$


and consequently for $\mu_{g}$-almost every $y$

$$
\lim _{n \rightarrow \infty} \frac{1}{n} \sum_{i=0}^{n-1} \varepsilon_{i}\left(\pi^{-1}(y)\right) \log \left|g^{\prime}\left(\sigma_{g}^{i} y\right)\right|=\int_{K(g)} \log \left|g^{\prime}\right| d \mu_{g}=\gamma(g) .
$$

Since the image under $\pi$ of $\mu_{f}$ is $\mu_{g}$, we obtain the statement of the lemma.

We now prove theorem 3. Assume that $f \in W^{s}(g)$. Set $\beta(g)=\log (1 / a(g))+\gamma(g)$. By lemma 8 , for $\mu_{f}$-a.e. $x$, all $\varepsilon>0$ and $m$ large enough we have

$$
\mu_{f}([x-\exp (-m(\beta(g)-\varepsilon)), x+\exp (-m(\beta(g)-\varepsilon))]) \geq 2^{-m} .
$$

Thus,

$$
\limsup _{\eta \rightarrow 0} \frac{\log \mu_{f}([x-\eta, x+\eta])}{\log \eta} \leq \frac{\log 2}{\beta(g)-\varepsilon} \quad \text { for } \mu_{f} \text {-a.e. } x \text {. }
$$

Fix $\varepsilon>0$. Set $\delta_{n}=\exp (-n(\beta(g)+\varepsilon))$. Denote by $Z_{n}$ the union of components of $J_{n}(f)$ whose lengths are not larger than $\delta_{n}$ and set $M_{n}=\bigcup_{k \geq n} Z_{k}$. By lemma 8, $\lim _{n \rightarrow \infty} \mu_{f}\left(M_{n}\right)=0$.

Fix $n_{0}$ such that $\mu_{f}\left(M_{n_{0}}\right) \leq \varepsilon$. Since $\mu_{f}$-a.e. point of $S \backslash M_{n_{0}}$ is a point of density of $S \backslash M_{n_{0}}$, and $\lim _{n \rightarrow \infty} \delta_{n}=0$, we have for $\mu_{f}$-a.e. point $x$ in $S \backslash M_{n_{0}}$,

$$
\lim _{n} \frac{\mu\left(\left(S \backslash M_{n_{0}}\right) \cap\left[x-\delta_{n}, x+\delta_{n}\right]\right)}{\mu\left(\left[x-\delta_{n}, x+\delta_{n}\right]\right)}=1 .
$$

By definition if $n \geq n_{0}$ there are at most 3 components of $J_{n}(f)$ intersecting $\left(S \backslash M_{n_{0}}\right) \cap$ $\left[x-\delta_{n}, x+\delta_{n}\right]$ and thus $\mu\left(\left(S \backslash M_{n_{0}}\right) \cap\left[x-\delta_{n}, x+\delta_{n}\right]\right) \leq 3.2^{-n}$ if $n \geq n_{0}$. Therefore on a set of measure $\geq 1-\varepsilon$ we have

$$
\liminf _{\eta \rightarrow 0} \frac{\log \mu_{f}([x-\eta, x+\eta])}{\log \eta} \geq \frac{\log 2}{\beta(g)+\varepsilon} .
$$

Theorem 3 follows from (6) and the above inequality by letting $\varepsilon$ go to zero.

Finally let us prove corollary 3 . Since $a(g)>0$ and $\left|g^{\prime}\right|$ is bounded, $\log (1 / a(g))+$ $\gamma(\mathrm{g})$ is finite and thus $\operatorname{dim} \mu_{f}>0$. If we had $\int \log \left|f^{\prime}\right| d \mu_{f}>0$, as $h_{\mu}(f)=0$, we should have, by formula (*), dim $\mu_{f}=0$. Therefore $\int \log \left|f^{\prime}\right| d \mu_{f}=0$, and also $\int \log \left|g^{\prime}\right| d \mu_{\mathrm{g}}=$ 0 .

\section{Appendix: proof of (*)}

Relation (*) in the uniformly hyperbolic case is an easy consequence of the ShannonMcMillan-Breiman theorem (see [1]). In the non-uniformly hyperbolic case, partial results with a weaker notion of convergence were given in [9]. The arguments from [9] also give relation ( $*$ ) for a certain class of maps, as we indicate briefly now. We shall consider a $C^{1}-\operatorname{map} f:[0,1] \rightarrow[0,1]$ and an invariant ergodic probability measure $\mu$. We denote by $h_{\mu}(f)$ the metric entropy of the system $([0,1], \mu, f)$, and by $\lambda_{\mu}(f)$ the Lyapunov exponent $\lambda_{\mu}(f)=\max \left(\int \log \left|f^{\prime}\right| d \mu, 0\right)$.

Proposition A1. Consider a $C^{1}$-map $f:[0,1] \rightarrow[0,1]$ and $\mu$ an invariant ergodic probability measure, then for $\mu$-a.e. $x$,

$$
h_{\mu}(f) \leq \lambda_{\mu}(f) \cdot \liminf _{\eta \rightarrow 0} \frac{\log \mu([x-\eta, x+\eta])}{\log \eta}
$$


Proof. By Ruelle's formula [12], $h_{\mu}(f) \leq \lambda_{\mu}(f)$ and we may thus suppose $\lambda_{\mu}(f)>0$. A result of Brin and Katok [2] says that for $\mu$-a.e. $x$,

$$
\lim _{\delta \rightarrow 0} \liminf _{n \rightarrow \infty}-\frac{1}{n} \log \mu(B(x, n, \delta))=h_{\mu}(f),
$$

where $B(x, n, \delta)$ is the set of $y$ in $[0,1]$ such that $\left|f^{i} y-f^{i} x\right| \leq \delta$ for $i=0,1, \ldots, n-1$. Therefore, we have only to show that for any $\delta>0$, there exists a sequence of functions $\left(\beta_{n}(x)\right)_{n=1}^{\infty}$ such that $\left[x-\beta_{n}(x), x+\beta_{n}(x)\right] \subset B(x, n, \delta)$ and

$$
\limsup _{n}-\frac{1}{n} \log \beta_{n}(x) \leq \lambda_{\mu}(f)+\delta \quad \mu \text {-a.e. }
$$

Fix $\delta>0$ and choose $r>0$ such that

$$
\int \log \left(\max \left(\left|f^{\prime}\right|, r\right)\right) \leq \lambda_{\mu}(f)+\delta / 2
$$

Write $\left|f^{\prime}\right|_{r}=\max \left(\left|f^{\prime}\right|, r\right)$. Since $\log \left|f^{\prime}\right|_{r}$ is a uniformly continuous function, we choose $\varepsilon>0$ such that if $|x-y| \leq \varepsilon$, then

$$
\left|f^{\prime}(x)\right|_{r} \leq\left|f^{\prime}(y)\right|_{r} \exp \delta / 2
$$

Set for $n \geq 1$,

$$
\beta_{n}(x)=\frac{\exp (-n \delta / 2) \cdot \min (\delta, \varepsilon)}{\max \left(1, \max _{1 \leq i \leq n} \prod_{k=0}^{i-1}\left|f^{\prime}\left(f^{k} x\right)\right|_{r}\right)} .
$$

Then for $\mu$-a.e. $x$

$$
\begin{aligned}
\lim _{n}-\frac{1}{n} \log \beta_{n}(x) & =\frac{\delta}{2}+\lim _{n} \frac{1}{n} \max \left(0, \max _{1 \leq i \leq n} \sum_{k=0}^{i-1} \log \left|f^{\prime}\left(f_{x}^{k}\right)\right|_{r}\right) \\
& =\frac{\delta}{2}+\max \left(0, \int \log \left|f^{\prime}\right|_{r} d \mu\right) \leq \lambda_{\mu}(f)+\delta .
\end{aligned}
$$

Besides, if $|x-y| \leq \beta_{n}(x)$, it is easy to check inductively on $i \leq n$ that $\left|f^{k} x-f^{k} y\right| \leq$ $\min (\delta, \varepsilon)$ for $k=0,1, \ldots, i-1$. The sequence $\left(\beta_{n}(x)\right)_{n=1}^{\infty}$ has the required properties.

Proposition A2. Consider a $C^{1}-$ map $f:[0,1] \rightarrow[0,1]$ and suppose that there exists a partition $Q$ of $[0,1]$ into intervals such that the functions $f$ and $f^{\prime}$ are monotonic on each element of $Q$. Consider an invariant ergodic probability measure $\mu$ such that $H_{\mu}(Q)<+\infty$. Then for $\mu$-a.e. $x$

$$
\lambda_{\mu}(f) \cdot \lim \sup _{\eta \rightarrow 0} \frac{\log \mu([x-\eta, x+\eta])}{\log \eta} \leq h_{\mu}(f) .
$$

Proof. We may suppose $\lambda_{\mu}(f)>0$. Fix $\varepsilon>0$. Since the function $\log \left|f^{\prime}\right|$ is integrable, the partition $P=\left\{P_{m}, m \in \mathbb{Z}\right\}$ has finite entropy, where $P_{m}=\left\{x|\exp -m \varepsilon \leq| f^{\prime} \mid<\right.$ $\exp -(m-1) \varepsilon\}$.

Denote by $p(n, x)$ the atom of the partition $\bigvee_{i=0}^{n-1} f^{-i}(P \vee Q)$ containing $x$. By the Shannon-McMillan-Breiman theorem, we have for $\mu$-a.e. $x$

$$
\lim _{n}-\frac{1}{n} \log \mu(p(n, x)) \leq h_{\mu}(f) \text {. }
$$


Since $p(n, x)$ is an interval where $f^{n}$ is monotonic and where $\left|\left(f^{n}\right)^{\prime}\right| \geq$ $\left|\left(f^{n}\right)^{\prime}(x)\right| \exp (-n \varepsilon)$, we have $|p(n, x)| \leq \exp (n \varepsilon) /\left|\left(f^{n}\right)^{\prime}(x)\right|$. Since for $\mu$-a.e. $x$ $\lim _{n}(1 / n) \log \left|\left(f^{n}\right)^{\prime}(x)\right|=\lambda_{\mu}(f)$, the inequality follows.

Corollary. Consider a $C^{1}$-map $f:[0,1] \rightarrow[0,1]$ such that $f$ and $f^{\prime}$ are piecewise monotonic and an invariant ergodic probability measure $\mu$. Suppose that $\lambda_{\mu}(f)>0$. Then for $\mu$-a.e. $x$ :

$$
\lim _{\eta \rightarrow 0} \frac{\log \mu([x-\eta, x+\eta])}{\log \eta}=\frac{h_{\mu}(f)}{\lambda_{\mu}(f)}
$$

\section{REFERENCES}

[1] P. Billingsley. Ergodic Theory and Information. J. Wiley and Sons, 1965.

[2] M. Brin \& A. Katok. On local entropy. In Geometric Dynamics. Lect. Notes in Math. 1007 Springer (1983).

[3] M. Campanino \& H. Epstein. On the existence of Feigenbaum's fixed point. Commun. Math. Phys. 79 (1981), 261-302.

[4] P. Collet \& J.-P. Eckmann. Iterated maps on the interval as dynamical systems. Progress in Physics, Birkhäuser: Boston, 1980.

[5] P. Collet, J.-P. Eckmann \& O. E. Lanford. Universal properties of maps on an interval. Commun. Math. Phys. 76 1980, 211-254.

[6] P. Grassberger. On the Hausdorff dimension of fractal attractors. Journal of Stat. Phys. 26 (1981), 173-179.

[7] K. M. Khanin, J. G. Sinai \& H. B. Vul. Feigenbaum universality and thermodynamical formalism. Uspehi 39; 3 (1984).

[8] O. E. Lanford. A computer-assisted proof of the Feigenbaum conjectures. Bull. Amer. Math. Soc. (New Series) 6 (1982), 427-434.

[9] F. Ledrappier. Some relations between dimension and Lyapounov exponents. Commun. Math. Phys. 81 (1981), 229-238.

[10] M. Misiurewicz. Structure of mappings of an interval with zero entropy. Publ. Math. IHES 53 (1981), 5-16.

[11] M. Misiurewicz. Attracting Cantor set of positive measure for a $C^{\infty}$ map of an interval. Ergod. Th. \& Dynam. Sys. 2 (1982), 405-415.

[12] D. Ruelle. An inequality for the entropy of differentiable maps. Bol. Soc. Bras. Mat. 9 (1978), 83-87. 